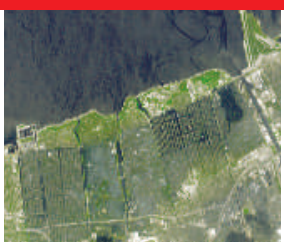

\title{
Brain imaging ready to detect terrorists, say neuroscientists
}

Brain-imaging techniques that reveal when a person is lying are now reliable enough to identify criminals, claim researchers.

Critics maintain that the technique will never be useful for such investigations, arguing that, as with traditional polygraph detectors, liars could learn to fool the tests. And researchers in the field have previously admitted that the approach needs more work. But neuroscientists from the University of Pennsylvania School of Medicine in Philadelphia have now told Nature that they believe their test is ready for real-life scenarios.

Daniel Langleben and his colleagues use functional magnetic resonance imaging (fMRI) to track people's brains when they lie and tell the truth. By analysing brain activity during both scenarios, they have developed an algorithm that can detect lies from truth with 99\% accuracy.

Name game: some say the word 'planet' is used too widely for it to be a useful definition.

Williams hopes to send a final version of the proposal to the IAU within two weeks, after the team has reviewed it. But whereas the broad definition of planetary objects is uncontroversial, at least one member plans to dispute the names for subtypes. "I don't believe we should classify planetary types by location," says Alan Stern of the Southwest Research Institute in Boulder, Colorado. "We should use properties of the objects as a guide."

UB313 and Pluto would be better known as "ice dwarfs", Stern suggests, because such a definition "tells us more about the objects". He points out that stars are classified by their physical properties, not their location.

If the group can reach a consensus, it will be up to the IAU's executive committee to decide whether to accept the proposal. But will the public and scientists then change the names they use for Mercury and Mars? "Old habits die hard," says Jacqueline Mitton, an author of popular astronomy books based in Cambridge, UK. She points out that some astrophysicists still describe stars as either 'early' or 'late' types, terminology that was officially abandoned around 50 years ago. "Committees can make pronouncements, but they can't always change things," she adds. "It will take a very long time."

Jim Giles the screen matched one of theirs. They were asked to be honest about having one of the cards and to lie about having the other.

Langleben has previously warned that fMRI is a research tool, not a way to spot liars. But the latest research has changed his tune. "We can't say whether this person will one day use a bomb," he says. "But we can use fMRI to find concealed information. We can ask: is $\mathrm{X}$ involved in terrorist organization Y?"

The main advance is being able to distinguish lies from truthful statements in a given individual. Although previously scientists could see how the brain lit up when people lied, results were based on the averaged brain activity of a group of people and did not look at individual fibs for each person. "Now we can tell when an individual lies on a specific question," says Gur. "This is a major step forward."

Critics argue that lab experiments do not equate to real-life situations. Getting a reward for concealing a lie is not the same thing as losing your job or getting a criminal conviction for being found out, which is a far more likely consequence, says Jennifer Vendemia, an expert in lie-detection research at the University of South Carolina, Columbia. "There is nothing you can do in the lab that would mimic job loss, the death penalty, or public humiliation.”

But the biggest concerns about using fMRI to detect lies, says Vendemia, are over ethical issues, such as whether individuals have the right to keep their thoughts private.

Critics and researchers agree that more funding is needed to standardize the method and iron out ethical concerns before the approach is used routinely. The team's next step is to expand its studies to include women, people of different cultures, and psychopaths. Jennifer Wild
Under fire: used properly, new brain-imaging techniques might assist in investigations of suspects. 